Bull. Austral. Math. Soc.

VOL. 62 (2000) [297-301]

\title{
ON SIMPLE, PRIMITIVE AND PRIME RINGS RELATIVE TO A TORSION THEORY
}

\author{
JOHN DAUNS AND YiQIANG Zhou
}

\begin{abstract}
For a hereditary torsion theory $\tau$, we show by examples that the concepts of $\tau$-Artinian $\tau$-simple, $\tau$-Artinian $\tau$-primitive, $\tau$-Artinian $\tau$-prime, $\tau$-Artinian $\tau$-semiprimitive, and $\tau$-Artinian $\tau$-semiprime rings are different from each other and thus answer a question raised by Bland in his book [Topics in Torsion Theory, (Mathematical Research, 103, Wiley-VCH, 1998)]. The example of a $\tau$-Artinian $\tau$-primitive ring which is not $\tau$-simple here appears to be a counter-example to a result of Bland in the same publication.
\end{abstract}

\section{INTRODUCTION}

Throughout, $R$ is an associative ring with identity, modules will be unitary right $R$-modules, and $\tau=\left(\mathcal{T}_{\tau}, \mathcal{F}_{\tau}\right)$ is a hereditary torsion theory on Mod- $R$, the category of all right $R$-modules. The following concepts can be found in [1]: A nonzero module $M$ is called $\tau$-simple if $M \in \mathcal{F}_{\tau}$ and $M / N \in \mathcal{T}_{\tau}$ for any nonzero submodule $N$ of $M ; J_{\tau}(R)$ is defined to be the intersection of all those right ideals $I$ of $R$ such that $R / I$ is $\tau$-simple; the ring $R$ is called $\tau$-Artinian if any descending chain $I_{1} \supseteq I_{2} \supseteq \cdots \supseteq I_{n} \supseteq \cdots$ of right ideals of $R$ with all $R / I_{n} \in \mathcal{F}_{\tau}$ terminates; $R$ is called $\tau$-primitive if the right annihilator of a cyclic $\tau$-simple $R$-module is $0 ; R$ is said to be $\tau$-semiprimitive if $J_{\tau}(R)=0$; a two-sided ideal $A$ of $R$ is said to be completely $\tau$-pure if $M /(M A) \in \mathcal{F}_{\tau}$ for any $M \in \mathcal{F}_{\tau} ; R$ is defined to be $\tau$-prime if whenever $A B=0$ for completely $\tau$-pure ideals $A$ and $B$ we have $A=0$ or $B=0 ; R$ is called $\tau$-semiprime if $A^{2}=0$ always implies $A=0$ for any completely $\tau$-pure ideal $A$; finally $R$ is called a $\tau$-simple ring if $R \in \mathcal{F}_{\tau}$ and whenever $R / I \in \mathcal{F}_{\tau}$ for an ideal $I$ of $R$ we have $I=0$ or $I=R$. The following ring implications were proved by Bland in [1]: $\tau$-simple $\Rightarrow \tau$-primitive $\Rightarrow \tau$-prime $\Rightarrow \tau$-semiprime and $\tau$-primitive $\Rightarrow \tau$-semiprimitive $\Rightarrow \tau$-semiprime. It was claimed in [1, Proposition 6.1.17] that any $\tau$-Artinian $\tau$-primitive ring is $\tau$-simple and it was then asked what other implications given above reverse under the assumption that $R$ is $\tau$-Artinian (see [1, p.142]).

Received 31st January, 2000

The research of the second author was supported by the NSERC grant OGP0194196. The first author thanks the hospitality of Memorial University during a research visit paid by the above grant.

Copyright Clearance Centre, Inc. Serial-fee code: 0004-9727/00 \$A2.00+0.00. 
In this short paper, we first give a counter-example to the result of Bland that any $\tau$-Artinian $\tau$-primitive ring is $\tau$-simple, and then answer Bland's question by giving examples of the following: A $\tau$-Artinian $\tau$-semiprimitive ring which is not $\tau$-prime; a $\tau$-Artinian $\tau$-prime ring which is not $\tau$-primitive; a $\tau$-Artinian $\tau$-semiprime ring which is neither $\tau$-semiprimitive nor $\tau$-prime.

\section{A $\tau$-ARTINIAN $\tau$-PRIMITIVE RING NEED NOT BE $\tau$-SIMPLE}

There exists a ring $R$ and a hereditary torsion theory $\tau$ such that $R$ is a $\tau$-Artinian $\tau$-primitive ring, but $R$ is not a $\tau$-simple ring. For a module $M_{R}, M^{\perp}$ is the annihilator of $M$ in $R$.

Example 1.1. Let $R=\left(\begin{array}{cc}F & F \\ 0 & F\end{array}\right)$ where $F$ is a field, $I=\left(\begin{array}{cc}F & F \\ 0 & 0\end{array}\right)$ and $J=\left(\begin{array}{ll}0 & F \\ 0 & 0\end{array}\right)$. Then $R \supset I \supset J \supset 0$ is a composition series of right ideals of $R$, so $R$ is right Artinian. Let $M_{R}=I$ and $N_{R}=J$. Then $M_{R}$ is cyclic faithful and $N_{R}$ is simple. Let $\mathcal{K}=\{X \in$ Mod- $R: \forall 0 \neq Y \subseteq X, N \hookrightarrow Y\}$. Then $\mathcal{K}$ is a natural class, that is, $\mathcal{K}$ is closed under submodules, direct sums, injective hulls and isomorphic copies. Since $R$ is right Artinian, $R$ has DCC on $\left\{L \subseteq R_{R}: R / L \in \mathcal{K}\right\}$. By [2, Proposition 21], $\mathcal{K}$ is a hereditary torsionfree class. Let $\tau$ be the hereditary torsion theory such that $\mathcal{F}_{\tau}=\mathcal{K}$. Then $R$ is $\tau$-Artinian. Clearly, $M \in \mathcal{K}$. Note that $N$ is the only non-trivial submodule of $M$ and $M / N \not \nexists N$. So, $M / N \notin \mathcal{K}$. This shows that $M$ is a $\tau$-simple module. So, $R$ is a $\tau$-primitive ring.

Note that $I$ is a two-sided ideal of $R$ and $(R / I)_{R} \cong N \in \mathcal{K}$. If $R$ is a $\tau$-simple ring, then it must be that $I=0$ or $I=R$, a contradiction.

In the above example, $M$ is a faithful cyclic $\tau$-simple $R$-module and $N$ is a cyclic $\tau$-simple $R$-module with $N^{\perp}=I \neq 0$. So, for a $\tau$-Artinian $\tau$-primitive ring $R$, the annihilator of some cyclic $\tau$-simple $R$-module may be non-zero. It seems that the incorrect statement that "the annihilator of each cyclic $\tau$-simple $R$-module over the $\tau$-Artinian $\tau$ primitive ring $R$ is zero" has been used in the proof of [1, Proposition 6.1.17] (see [1, line -6, p.142]).

\section{RESPONSE TO BLAND'S QUESTION}

As a response to Bland's question above, we give the following examples.

EXAMPLE 2.1. A $\tau$-Artinian $\tau$-semiprimitive ring which is not $\tau$-prime: Let $R$ be an Artinian semisimple ring, but one that is not simple. Let $\tau=\left(\mathcal{T}_{\tau}, \mathcal{F}_{\tau}\right)$ be the hereditary torsion theory in which every $R$-module is $\tau$-torsionfree, that is, $\mathcal{T}_{\tau}=\{(0)\}$ and $\mathcal{F}_{\tau}=$ Mod- $R$. Then every ideal of $R$ is completely $\tau$-pure. Since $R$ is semisimple but not simple, there exist nonzero ideals $A$ and $B$ such that $A B=0$. Thus, $R$ is not $\tau$-prime. But, clearly, $R$ is $\tau$-Artinian $\tau$-semiprime. We can further prove $R$ is $\tau$-semiprimitive. By the definition of $\tau$, an $R$-module is $\tau$-simple if and only if it is simple. Thus, for a right 
ideal $I$ of $R, R / I$ is $\tau$-simple if and only if $R / I$ is simple if and only if $I$ is a maximal right ideal. It follows that $J_{\tau}(R)$ is equal to the Jacobson radical $J(R)$ of $R$. But, clearly, $J(R)=0$.

The next two examples give $\tau$-Artinian $\tau$-semiprime rings which are neither $\tau$-prime nor $\tau$-semiprimitive.

EXAMPLE 2.2. Let $R$ be an Artinian semisimple ring with two simple $R$-modules, say $M$ and $N$, up to isomorphism. Let $\tau=\left(\mathcal{T}_{\tau}, \mathcal{F}_{\tau}\right)$ be the hereditary torsion theory cogenerated by $M$, that is, $X \in \mathcal{F}_{\tau}$ if and only if $X \hookrightarrow E(M)^{I}$ for some index set $I$, where $E(M)$ is the injective hull of $M$. Note that $R$ is Artinian semisimple and every $R$-module is injective. So, $X \in \mathcal{F}_{\tau}$ if and only if $X \cong M^{(J)}$ for some index set $J$. Then an $R$-module $X$ is $\tau$-simple if and only if $X \cong M$, and thus $X^{\perp}=M^{\perp}$. By [1, Proposition 2.2.8], $J_{\tau}(R)$ is the intersection of the annihilators of all cyclic $\tau$-simple $R$-modules. It follows that $J_{\tau}(R)=M^{\perp}$. By the assumption on $R, M^{\perp}$ is a nonzero proper ideal of $R$. So, $R$ is not $\tau$-semiprimitive. But, it is easy to see that $R$ is $\tau$-Artinian $\tau$-semiprime. To see that $R$ is not $\tau$-prime, note that $X \in \mathcal{F}_{\tau}$ if and only if $X \cong M^{(I)}$ for some index set $I$, and in this case, for any ideal $A$ of $R, X /(X A) \cong M^{(J)} \in \mathcal{F}_{\tau}$ for some index set $J$. This means that every ideal of $R$ is completely $\tau$-pure. By the assumptions of $R, R$ is not prime and so $R$ is not $\tau$-prime.

EXAMPLE 2.3. Let $Q=\prod_{i=1}^{\infty} F_{i}$, where $F_{i}=\mathbb{Z}_{2}=\{\overline{0}, \overline{1}\}$, be the direct product of rings $F_{i}, R$ the subring of $Q$ generated by $\bigoplus_{i=1}^{\infty} F_{i}$ and $1_{Q}$. Let $M=R /\left(\bigoplus_{i=1}^{\infty} F_{i}\right)$. Note that $M$ is an injective $R$-module. Let $\tau$ be the hereditary torsion theory cogenerated by $M$. Then $X \in \mathcal{F}_{\tau}$ if and only if $X \hookrightarrow M^{K}$ for some index set $K$. Thus, for a right ideal $I$ of $R, R / I$ is $\tau$-torsionfree if and only if $I=\bigoplus_{i=1}^{\infty} F_{i}$ or $I=R$. So, $R$ is $\tau$-Artinian. And a cyclic module $N$ is $\tau$-simple if and only if $N \stackrel{i=1}{=} M$, so, in this case, $N^{\perp}=M^{\perp}=\bigoplus_{i=1}^{\infty} F_{i} \neq 0$. Thus, $J_{\tau}(R)=$ the intersection of the annihilators of all cyclic $\tau$-simple $R$-modules $=\bigoplus_{i=1}^{\infty} F_{i} \neq 0$. Thus, $R$ is not $\tau$-semiprimitive. The above discussion shows that $\bigoplus_{i=1}^{\infty} F_{i} \subseteq X^{\perp}$ for every $\tau$-torsion free $R$-module $X$. It follows that every ideal of $R$ contained in $\bigoplus_{i=1}^{\infty} F_{i}$ is a completely $\tau$-pure ideal. This implies that $R$ is not $\tau$-prime. Since $R$ is semiprime, $R$ is $\tau$-semiprime.

EXAMPLE 2.4. A $\tau$-Artinian $\tau$-prime ring which is not $\tau$-primitive: Let $R$ be a prime ring but not a right primitive ring. Let $\tau=\left(\mathcal{T}_{\tau}, \mathcal{F}_{\tau}\right)$ be the hereditary torsion theory such that every $R$-module is a $\tau$-torsion module, that is, $\mathcal{T}_{\tau}=\operatorname{Mod}-R$ and $\mathcal{F}_{\tau}=\{(0)\}$. Then for any right ideal $I$ of $R, R / I$ is $\tau$-torsion free if and only if $I=R$. So, $R$ is $\tau$-Artinian and $\tau$-prime. Since the zero module ( 0 ) is the only $\tau$-torsion free module, there does not exist any $\tau$-simple module. So, there does not exist a $\tau$-simple module whose annihilator is zero. Therefore, $R$ is not $\tau$-primitive. 
Note that Example 2.4 is not desirable for Bland's question because it was assumed in $[1$, Section 6.1,p.135] that $\tau$ is a torsion theory on Mod- $R$ such that $\tau$-simple $R$ modules exist. So, the remaining question is the following: Does there exist a ring $R$ and a hereditary torsion theory $\tau$ on Mod- $R$ such that $\tau$-simple $R$-modules exist, $R$ is $\tau$-Artinian $\tau$-prime, but $R$ is not $\tau$-primitive ? The answer is "Yes", as shown by the next example.

EXAMPLE 2.5. Let $R$ be any ring with nonzero right ideals $I$ and $J, I \cap J=0$, satisfying the following properties:

(1) $I^{2} \neq I$ and $a b=b a$ for all $a, b \in I$;

(2) for any $r \in R \backslash J, 0 \neq r s \in I$ for some $s \in R$, and dually for any $r \in R \backslash I$, $0 \neq r t \in J$ for some $t \in R$;

(3) for any $0 \neq L \subseteq I, L^{\perp}=J$, and dually for any $0 \neq P \subseteq J, P^{\perp}=I$;

(4) lastly, $R /(I \oplus J)$ satisfies the $\mathrm{DCC}$ on right submodules.

Let $M_{R}=I$ and $\mathcal{K}=\{X \in$ Mod- $R: \forall 0 \neq Y \subseteq X, \exists 0 \neq Z \subseteq Y$ such that $Z \hookrightarrow M\}$. Then $\mathcal{K}$ is a natural class, that is, $\mathcal{K}$ is closed under submodules, direct sums, injective hulls and isomorphic copies. Observe that

$$
\text { for any } 0 \neq X \in \mathcal{K} \text {, and any } 0 \neq m \in I, X m \neq 0 \text { by (3). }
$$

Let $R / K \in \mathcal{K}$. Then $J I=0$ by (3) with $P=J$. Hence $[(J+K) / K] I=\overline{0}$. By $(*)$, $J \subseteq K$. Thus, $K=J$ or $K \supset J$. Suppose that $K \supset J$. Let $r \in K \backslash J$. Then by (2), $0 \neq m=r s \in I$. But then $I m=m I \subseteq I \cap K$ by (1). Thus $[I /(I \cap K)] m=\overline{0}$ and $I /(I \cap K) \cong(I+K) / K \in \mathcal{K}$, and by $(*), I \subseteq K$. Therefore $I \oplus J \subseteq K$. So, we have proved that if $R / K \in \mathcal{K}$ then $K=J$ or $K \supseteq I \oplus J$. In view of (4), $R$ has DCC on $\left\{L \subseteq R_{R}: R / L \in \mathcal{K}\right\}$. By [2, Proposition 21], $\mathcal{K}$ is a hereditary torsionfree class. Let $\tau=\left(\mathcal{T}_{\tau}, \mathcal{F}_{\tau}\right)$ be the hereditary torsion theory such that $\mathcal{F}_{\tau}=\mathcal{K}$. Then $R$ is $\tau$-Artinian. Clearly, $I \in \mathcal{F}_{\tau}$. If $0 \neq L \subset I$, then $(I / L) m=\overline{0}$ for $0 \neq m \in L$ (by (1)), so $I / L \notin \mathcal{K}$. So, $I$ is a $\tau$-simple $R$-module. Therefore, $r$-simple $R$-modules exist. Note that $J_{R} \notin \mathcal{F}_{\tau}$ by $(*)$ and (3), and thus $R_{R} \notin \mathcal{F}_{\tau}$. It follows from [1, Corollary 6.1.4, p.136] that $R$ is not a $\tau$-primitive ring. To show that $R$ is $\tau$-prime, let $A \neq 0$ and $B \neq 0$ be two completely $\tau$ pure ideals of $R$ such that $A B=0$. Suppose first that $A \nsubseteq J$. Then for $a \in A \backslash J$, by (2), $0 \neq a r \in I$. Thus, $(I / I A)(a r)=\overline{0}$ and $I /(I A) \in \mathcal{K}$, and by $(*), I=I A \subseteq A$. But then $I B \subseteq A B=0$, and $B \subseteq J$ by (3). If $A=I$, then $I=I A \subseteq I^{2} \subset I$ is a contradiction. So, $I \subset A$. By (2), for $b \in A \backslash I, 0 \neq b t \in J$. Then $(b t R) B \subseteq A B=0$ implies that $B \subseteq I$ by (3). It follows that $B \subseteq I \cap J=0$. This contradiction shows that $A \subseteq J$. Consequently $A B=0$ implies that $B \subseteq I$ by (3). For $0 \neq m \in B \subseteq I,(I / I B) m=\overline{0}$ and $I / I B \in \mathcal{K}$. By (*), $I=I B$, and this implies that $I=I^{2}$, a contradiction. Thus, $R$ is $\tau$-prime.

Below are two examples where the ring $R$ with the right ideals $I$ and $J$ satisfies the conditions (1)-(4) in Example 2.5. 
1. Let $T=\mathbb{Z} \oplus \mathbb{Z}$ be the ring direct sum, and $R=(2 \mathbb{Z} \oplus 2 \mathbb{Z})+\mathbb{Z} 1_{T}$ be the subring of $T$ generated by $2 \mathbb{Z} \oplus 2 \mathbb{Z}$ and $1_{T}$. Let $I=2 \mathbb{Z} \oplus(0)$ and $J=(0) \oplus 2 \mathbb{Z}$. Then $I$ and $J$ are ideals of $R$ and $R /(I \oplus J)=R /(2 \mathbb{Z} \oplus 2 \mathbb{Z})=$ $\{\overline{(0,0)}, \overline{(1,1)}\} \cong \mathbb{Z}_{2}$.

2. Let $\mathbb{Z}_{2}=\mathbb{Z} / 2 \mathbb{Z}$ and $\mathbb{Z}_{2}[t]$ be the polynomial ring. Let $T=\mathbb{Z}_{2}[t] \oplus \mathbb{Z}_{2}[t]$ be the ring direct sum, and $R$ the subring of $T$ generated by $t \mathbb{Z}_{2}[t] \oplus t \mathbb{Z}_{2}[t]$ and $1_{T}$. Let $I=t \mathbb{Z}_{2}[t] \oplus(0)$ and $J=(0) \oplus t \mathbb{Z}_{2}[t]$. Then $I, J$ are ideals of $R$ and $R /(I \oplus J) \cong \mathbb{Z}_{2}$.

\section{REFERENCES}

[1] P.E. Bland, Topics in torsion theory, Mathematical Research 103 (Wiley-VCH Verlag, Berlin, 1998).

[2] S.S. Page and Y. Zhou, 'On direct sums of injective modules and chain conditions', Canad. J. Math. 46 (1994), 634-647.

Tulane University

New Orleans LA 70118-5698

United States of America
Memorial University of Newfoundland

St.John's, NF AlC 5S7

Canada 